\title{
The fault simulation methods of hydraulic system based on AMESim
}

\author{
Shiming Liu ${ }^{1, a^{*}} \quad$ Guohui Zhang ${ }^{2, b}$ \\ ${ }^{1}$ The School of Applied Technology, University of Science and Technology Liaoning, Anshan, China \\ ${ }^{2}$ Dalian University of Technology, Master of mechanical, Class1502, Liaoning, Dalian, China \\ alsm686@sohu.com , ${ }^{\mathrm{b}} 412881526 @ q q . c o m$
}

Key words: fault simulation hydraulic pressure AMESim

Abstract: in this paper, the simulation method based on AMESim for the common fault of hydraulic system is discussed, two most basic fault simulation methods are summarized, namely parameter setting method and additional element method, and the detailed analysis of each method is given. At last these two methods are compared, their own application focus is given.

\section{Introduction}

Because of the closed feature, complexity and the overlap of the hydraulic system fault, it is not only time-consuming, expensive but also difficult to use the conventional method to study it. However simulation method can be used to solve the problem. AMESim is the software specially used in hydraulic / mechanical system modeling, simulation and dynamic analysis. The use of AMESim for hydraulic fault simulation can easily change mathematical physics model of the machine, electricity, liquid coupling system into visualized, modular simulation model. Besides, by modifying the parameters of the system to put in system fault information and to achieve the dynamic simulation of hydraulic system fault, which provides a reliable basis for fault diagnosis and prediction.

\section{Fault simulation basic method}

Through the application of AMESim software, and on the basis of summing up the experience of others, the use of AMESim software to simulate the hydraulic fault of the method is summarized as two basic methods: parameter setting method and additional element method.

\section{Parameter setting method}

Parameter setting method: after the establishment of accurate system simulation model, according to the different fault simulation projects, the normal model is put into the different fault parameters, so that the simulation model can be run under the condition of failure, thus the parameters of the system with fault can be obtained.

(1) The spring failure

Spring serves as the most common components of the hydraulic components, are widely used. the reversing valve, relief valve, pressure regulator valve of the hydraulic system and hydraulic cylinder It is very important to maintain the stability of spring elastic coefficient and other parameters. However, with the increase of the number of application, the elastic coefficient of spring will be changed gradually. For the actual hydraulic system, it is very difficult to judge this kind of change directly, but it can be used to simulate the elastic coefficient of spring in the simulation model, so as to establish the relationship of the performance of the spring and the failure phenomenon. Specific methods as shown in Fig. 1, the stiffness spring are used to simulate the constant degradation of the 
spring elastic coefficient in the parameter box. When the spring can be completely failed, stiffness coefficient can be set to a nonzero infinitesimal number for simulation.

(2) The fault of blocked hydraulic system

$70 \%$ of the failure in hydraulic system is caused by oil pollution, a large part of the fault caused by oil pollution is a variety of circuit plug, and the hydraulic components of the various damping hole and servo valve nozzle due to its small size, the oil is the most easy to be contaminated and blocked. Here we can control the size of the damping hole and the aperture of the servo valve to simulate the blockage fault, and through the setting of different degree of blockage to observe the failure phenomenon of different blockage. As shown in Fig. 2, we can simulate different blocking conditions by setting the flow rate of the aperture.

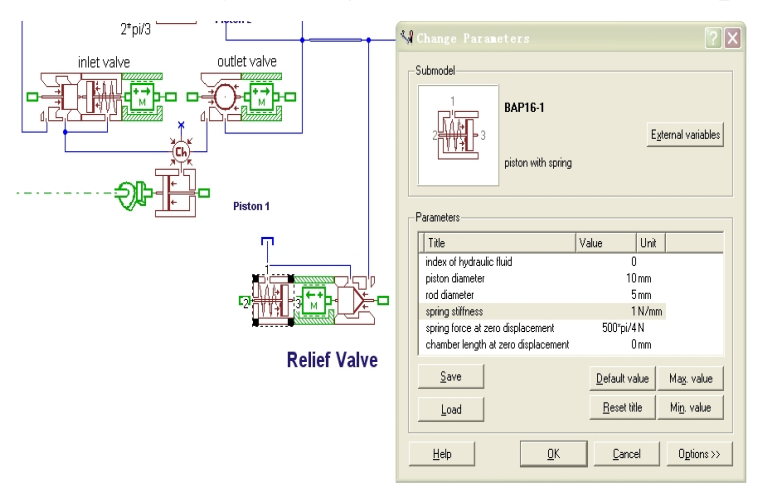

Fig. 1 spring parameter adjustment

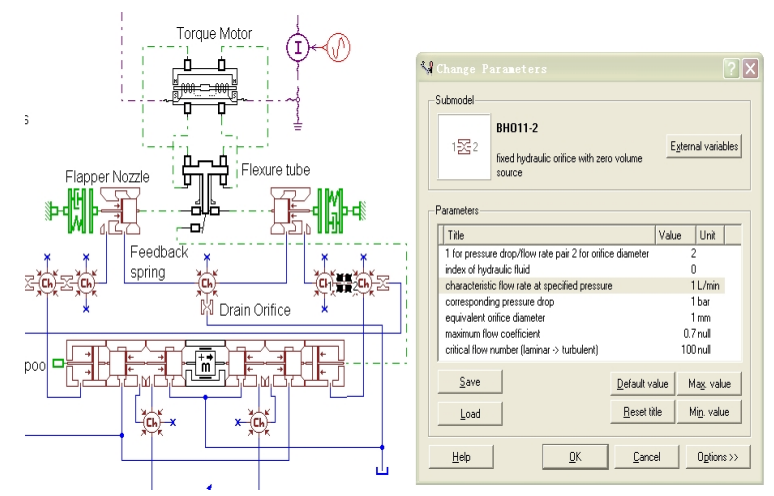

Fig. 2 damping aperture adjustment

(3) The hydraulic cylinder oil leakage and interface

Leakage is one of the most common failures in hydraulic system, the leakage of hydraulic cylinder is divided into internal leakage and external leakage. External can be found by observation, but the internal leakage has strong invisibility, and it is very difficult to find out leakage under medium degree without special inspection. Through fault simulation, we can find out the relationship between the hydraulic cylinder's internal leakage and hydraulic cylinder's response time and response speed, which provides a basis for judging the extent of the internal leakage of the hydraulic cylinder by readily observable phenomenon after For the hydraulic cylinder internal leakage, it can be adjusted by coefficient leakage to simulate different internal leakage, observe the hydraulic cylinder leakage state.

(4) The friction stuck fault

Because of the existence of various kinds of debris in the hydraulic oil, which results the pressure loss and the loss of components, even the loss of the components of the hydraulic system, and the influence of the system operation, so it is very important to simulate the failure of the hydraulic system in order to understand the cause of failure. For this kind of fault, we can simulate the hydraulic cylinder operation difficulty and the stuck failure of the hydraulic cylinder caused by oil contamination through adjusting the viscous friction coefficient and friction force Coulomb.

(5) The influence of varying oil properties of hydraulic system

When oil viscosity changes, it can be set different oil viscosity to simulate the condition. For example, under normal circumstances, the viscosity of the oil is $51 \mathrm{cP}$, it can be set separately in the different values of the normal oil viscosity lower or higher of normal value in the simulation.

When the oil is mixed with air, the system responds slowly, when a large amount of air included, the system will appear the shock oscillation, which is consistent with the theoretical analysis results 
of the oil volume elastic modulus decreased. Therefore, in the simulation, it can be simulated by reducing the elastic modulus of the oil volume. For example under normal circumstances, the bulk modulus of elasticity is $1700 \mathrm{MPa}$, which can be simulated by setting the number of numerical values below and above normal.

In addition, there are other similar types of failures, methods are the same, they are not elaborated here.

\section{additional element method}

Additional element method: in the condition of not affecting the operation function of the original hydraulic system, added one or several hydraulic components into the system, and through adjusting the parameters of these components to achieve the purpose of regulating the specific element parameters of the hydraulic system, to carry out the fault simulation of the specific components, thus to get the failure phenomenon of the hydraulic system.

(1) The internal leakage of hydraulic cylinder

Hydraulic cylinder leakage can cause the hydraulic cylinder creeping failure, the simulation can be a hydraulic cylinder parallel with a throttle valve, through setting the flow of the throttle valve to simulate the hydraulic cylinder leakage fault. Fig. 3 shows the paralleled internal oil circuit, by adjusting the flow rate of the throttle valve, to simulate different conditions of the hydraulic cylinder leakage.

(2) The leakage of oil interface

Due to the aging of the interface, its loose and other reasons, the hydraulic system oil circuit and its interface is often leaked, we can simulate the leakage through the external oil circuit to observe the leakage of the failure phenomenon. As shown in Fig. 3, adjusting the leakage of oil spill valve can control the size of the hydraulic cylinder interface leakage.

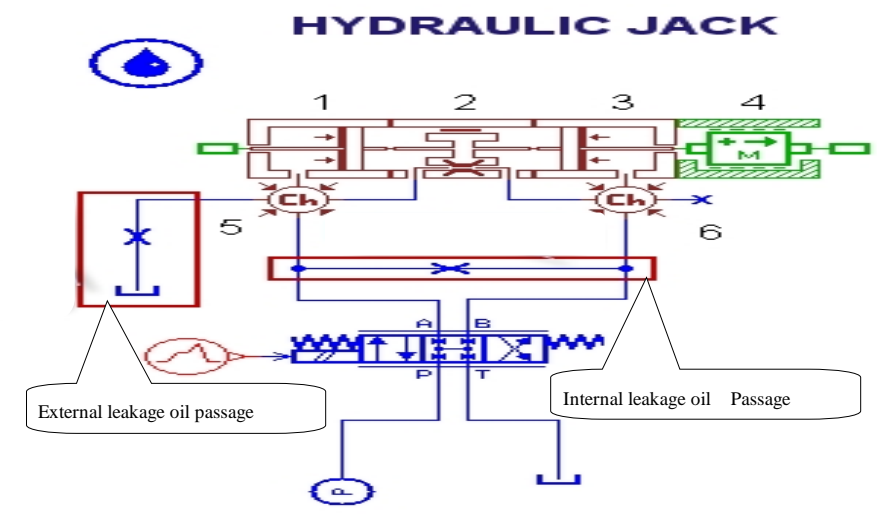

Fig. 3 additional internal and external leakage oil passage

\section{Application and analysis}

Faults in hydraulic system of certain equipment are leakage mainly, the reason is mainly leaks into the tubing, hydraulic valve and the cylinder caused by the loosening of system nut, screw and pipe joint. Here we take the lift cylinder as the research object, using the parameter setting method and the additional element method separately to simulate the influence of the leakage fault on the system operation In Fig. 4, the simulation model using the parameter setting method and the additional element method are respectively used in the box. The simulation parameters for the two methods are shown in Table 1. Fig. 5 shows the two kinds of methods to simulate the hydraulic cylinder leaks. Fig. 6 shows displacement of hydraulic cylinder piston in case of gradually increasing leakage. As can be seen from the figure, the results obtained of the two different fault 
simulation methods are basically the same, it can be seen as the leakage rate increases, the response time of the hydraulic cylinder piston is longer and longer, which is consistent with the actual situation. Although the simulation results of the two methods are basically the same, there are some differences: in the parameter setting method, the hydraulic cylinder leakage formula is $q_{\text {leak }}=\left(p_{11}-p_{22}\right) \bullet$ leak, its value is only related to the pressure and leakage coefficient of the two chamber; In additional element method, the leakage of hydraulic cylinder and the characteristics of leakage coefficient, pressure drop and hydraulic oil in the laminar flow state or the state of turbulence are all related. In Fig. 6, although the leakage of the two methods are the same, the reaction time is still a big difference between the two hydraulic cylinders. It can be seen that two kinds of simulation methods can be used to achieve different purposes. The parameter setting method can be used to study the relationship between the leakage rate and the reaction time in a big system; Additional element method can be used to study when hydraulic leakage occurs, to measure different leakage conditions accurately.

Table 1 simulation parameter settings

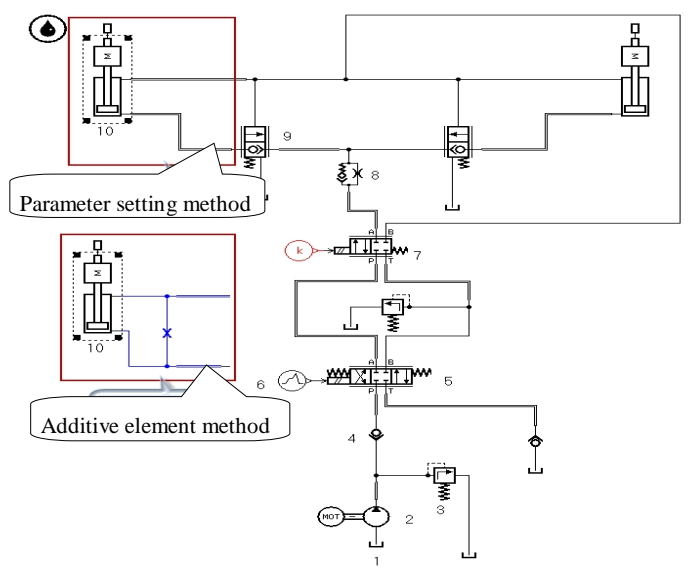

\begin{tabular}{|c|c|c|c|c|c|}
\hline Method & Parameter & 1 & 2 & 3 & 4 \\
\hline $\begin{array}{c}\text { Parameter } \\
\text { setting } \\
\text { method }\end{array}$ & $\begin{array}{c}\text { leakage } \\
\text { coefficient } \\
(\mathrm{L} / \mathrm{min} / \mathrm{bar})\end{array}$ & 0 & 0.03 & 0.06 & 0.09 \\
\hline $\begin{array}{c}\text { Additive } \\
\text { element } \\
\text { method }\end{array}$ & $\begin{array}{c}\text { Characteristic } \\
\text { flow }\end{array}$ & 0 & 0.61 & 1.18 & 1.76 \\
\cline { 2 - 6 } & $\begin{array}{c}\text { corresponding } \\
\text { pressure } \\
\text { drop(bar) }\end{array}$ & 28.24 & 28.27 & 28.16 & 28.24 \\
\hline
\end{tabular}

Fig. 4 fault simulation hydraulic system

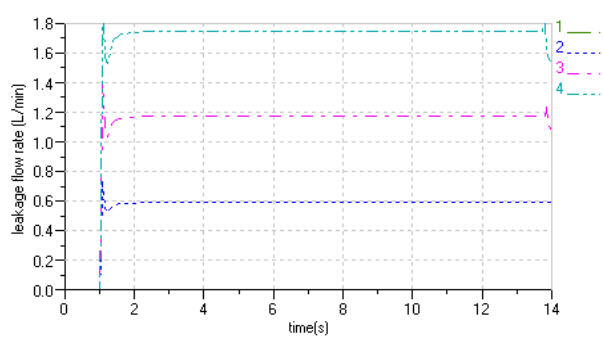

a)Parameter setting method

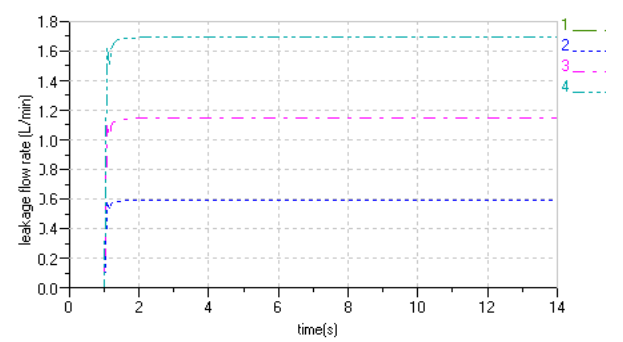

b)Aditional element method

Fig. 5 hydraulic cylinder leakage curve

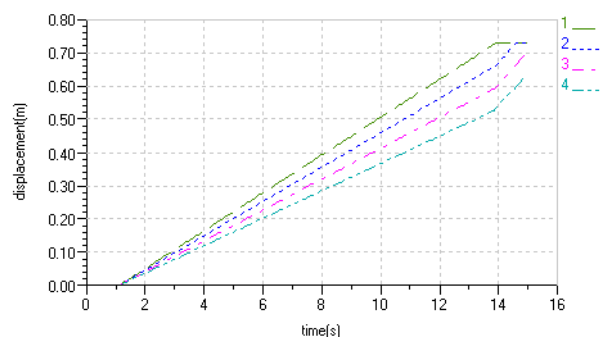

a)Prameter setting method

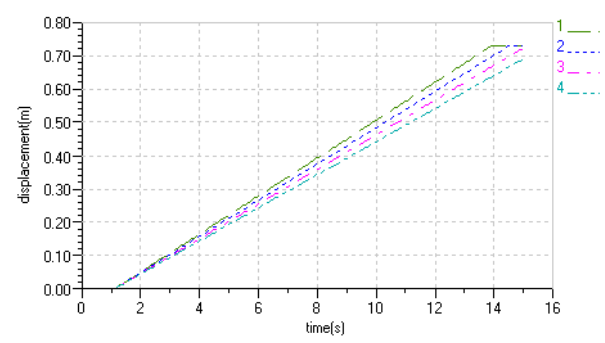

b)Aditional element method

Fig. 6 displacement response curve of piston 


\section{Conclusions}

Parameter setting method and additional element method can be easily used in fault simulation of hydraulic system. The two methods can be used to simulate most of the faults in hydraulic system, so as to understand the reason and the mode of faults of hydraulic system, which provides a reference for fault diagnosis and prediction.

\section{References}

[1] Kang Si .Research on fault diagnosis of electro hydraulic servo system based on AMESim simulation [D]. Wuhan .Wuhan University of Science and Technology.2009

[2] Dianyu Yang. Simulation Research on the hydraulic system of a missile launching guidance based on AMESim[D]. Shijiazhuang. The Ordnance Engineering Institute. 2010

[3] Guopeng Peng, Huang Haitao Peng. Hydraulic failure analysis and dynamic simulation of radar antenna [J]. Fire control radar technology, 2009, (2): 43 - 47

[4] Ning Hu, Zhen Chen. Reliability analysis of AT hydraulic control system based on AMESim [J]. Machine tools and hydraulic, 2011, (3): 145 - 147 